\title{
Investigación industrial sobre ahorro de energía en la fabricación de nuevos cementos compuestos españoles*
}

OFICEMEX: Grupo de Trabajo formado por:

II. AGLANELI, J. BARAGASO, F. GOMA, V. GONZALEZ VILA, R. LOPEZ SOLER,

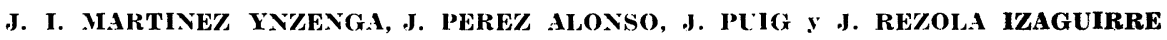

\section{R E S U M E N}

Se exponen en este trabajo los resultados de un estudio consistente en la preparación de varios cementos nuevos, en diversas fábricas de diferentes zonas del país, basada en la utilización de materiales de adición, tanto activos como fillers calizos, con objeto de contribuir al ahorro energético en la fabricación de cemento.

Los nuevos cementos fabricados se comparan con los producidos en la actualidad y se llega a la conclusión de que muchos de ellos permitirán sustanciales ahorros de fuel-oil, sin merma sensible, en la mayoría de los casos - y con mejora en otros- de la calidad $\mathrm{y}$ del comportamiento de los cementos nuevos.

\section{INTRODUCCION}

Durante el desarollo y la evolución de la Industria Española del Cemento se ha llegado a conseguir un amplio conocimiento y experiencia en la utilización sucesiva de combustibles sólidos, líquidos y gaseosos, para volver recientemente al empleo progresivo del carbón, por razones evidentes.

El aprovechamiento de materiales como las puzolanas y las adiciones calizas, así como de subproductos y residuos industriales como las escorias siderúrgicas y las cenizas volantes de centrales termoeléctricas, ha sido un empeño constante por parte del Sector Industrial del Cemento.

El retorno al carbón y el aprovechamiento de materiales activos que no pasan por el horno han supuesto dos vías complementarias para ahorrar fuel-oil y, en general, energía, lo cual supone uno de los objetivos perseguidos hoy en todos los países.

* Trabajo presentado por el Prof. Dr. J. CALLEJA, del IETCC, en el Colloque International sur les Laitiers et les Ciments aux Ajouts, MONS (Bélgica), 7-11 de Septiembre de 1981. 
Esta ha sido la razón por la cual OFICEMEN (la Agrupación de Fabricantes de Cemento de España) ha cumpl:do el propósito recomendado por las autoridades españolas de ahorrar energía en la fabricación de cemento, sustituyendo una parte de clínker por los materiales y productos mencionados, con tal de que la calidad y el comportamiento de los cementos se mantenga dentro de límites suficientemente aceptables.

\section{SITUACION ANTERIOR}

\subsection{Tipos, clases y categorías de cementos}

Dentro del amplio campo de cementos incluidos en la actualidad en el Pliego de Cementos RC-75, sólo los cementos portland $\mathrm{P}$ y los cementos con hasta 20 por ciento de adiciones activas, PA, van a ser considerados en lo que sigue. Tampoco se tendrán en cuenta otras categorías que las de 450 y 350 -resistencias mínimas a compresión, en $\mathrm{kp} / \mathrm{cm}^{2}$ a 28 días, en probeta de mortero normal ISO-RILEM-CEMBUREAU-, dado que dichas categorías suponen aproximadamente el 95 por ciento del consumo total español de cemento.

\subsection{Ahorro de energía}

Los ahorros de energía en la clinkerización convencional no son ya tan sustanciales como lo fueron antaño. La razón es su caracter asintótico, de tal manera que exiguas mejoras en el ahorro requieren enormes gastos con poco o ningún provecho. Por otra parte, la clinkerización no convencional podría ser, a lo sumo, una solución a medio o largo plazo, pero mucho menos cómoda, económica e inmediata.

Por consiguiente, el ahorro de energía en la fabricación de cemento debe orientarse hacia la sustitución parcial de clínker por recursos naturales o subproductos consistentes en materiales activos.

\subsection{La influencia de la tobermorita en las resistencias}

La resistencia mecánica de la pasta de cemento es debida a las propiedades de los geles tobermoríticos formados en la hidratación de los silicatos del clínker, en la cual se forma también portlandita.

En las pastas de los cementos que contienen adiciones activas la tobermorita total es la suma de la procedente de la hidratac:ón directa de los silicatos - tobermorita primaria- y de la producida por reacción de la portlandita con los componentes ácidos de las adiciones -tobermorita secundaria-.

Además, la velocidad de formación de las tobermoritas, el tamaño de sus partículas y las distancias entre sus planos reticulares tienen una gran influencia sobre la resistencia de la pasta de cemento a plazos corto y medio. Todos estos factores se pueden modificar e incluso mejorar mediante la adición de pequeñas cantidades de filler calizo al clínker, ya que el filler puede actuar como agente dispersante que permite una hidratación más rápida y completa de los granos de cemento.

\subsection{La influencia de la relación agua/cemento en la resistencia}

Los fabricantes de cemento son conscientes del alto grado de infrautilización o de mala utilización a la que el material es frecuentemente sometido en la práctica por los usuarios. Ello es debido en gran medida al general incumplimiento de las especificaciones normales obligatorias para el hormigón y más particularmente de las que se refieren a la relación agua/cemento. 
El recurso a las relaciones agua/cemento más altas —en vez de utilizar aditivos para hormigón adecuados - es equivalente a probar la calidad del cemento en las condiciones más adversas. Por ejemplo, de acuerdo con el ensayo de mortero normal ISO-RILEM-CEMBUREAU, el cambio de la relación agua/cemento de 0,5 a 0,6 supone una pérdida de resistencia de cerca del 15 por ciento y del 12 por ciento respectivamente, para las categorías 450 y 350 , de tal manera que las categorías más altas son siempre las más perjudicadas.

\section{OBJETIVO PRINCIPAL}

En el presente trabajo se ha pretendido conseguir un doble fin: evaluar el ahorro de energía en la fabricación de los llamados "cementos-proyecto" y conseguir que tales cementos presenten propiedades y comportamientos semejantes a los de los llamados "cementos actuales", fabricados hasta la fecha en España. En tal sentido se ha programado y realizado la fabricación a escala industrial de los siguientes "cementos-proyecto":

i) "cementos-proyecto" obtenidos por adición de 5 por ciento de filier calizo a los "cementos actuales" que por ahora no contienen ninguna adición;

ii) "cementos-proyecto" obtenidos por aumento hasta un máximo de 35 por ciento, del máximo del 20 por ciento de adiciones activas en los "cementos actuales". Se consideró también la posibilidad de que una parte - hasta el 5 por ciento- en dicho 35 por ciento pudiera ser un filler calizo.

Los resultados obtenidos han sido publicados con detalle en un libro recientemente editado por OFICEMEN (1), del cual se distribuyeron ejemplares en el Coloquio Internacional de MONS (Bélgica). El presente trabajo es un resumen muy breve del contenido del libro mencionado.

\section{TRABAJO A ESCALA INDUSTRIAL}

\subsection{La fabricación de los cementos}

Se han tenido en cuenta en (1) los siguientes aspectos de la fabricación y del control de los cementos: selección y control de las materias primas, preparación de los crudos, clinkerización, molienda del cemento, consumo de energía, y ensayos físicos y químicos de los cementos.

La fabricación y los ensayos se llevaron a cabo en varias fábricas de cinco zonas cementeras españolas diferentes. El total de los 84 cementos preparados se distribuyeron así: 30 cementos P-450, 14 cementos P-350 y 40 cementos PA-350.

\subsection{Características de las materias primas}

Los datos completos sobre el análisis químico y la composición granulométrica de materias primas, crudos, yesos, calizas, escorias, puzolanas, cenizas volantes, clínkeres y cementos fueron expuestos con todo detalle en (1).

\subsubsection{Clinker}

La calidad y regularidad de los clínkeres producidos actualmente en España, y utilizados en la preparación de los cementos estudiados, ha sido tal que todos ellos presentaron diferencias muy pequeñas en su composición química, como quedó de manifiesto en (1). 


\subsubsection{Escorias granuladas de horno alto}

Las escorias básicas granuladas de horno alto utilizadas, procedentes de dos acerías españolas, cumplieron la condición química: $\left(\mathrm{CaO}+\mathrm{MgO}+\mathrm{Al}_{2} \mathrm{O}_{3}\right) / \mathrm{SiO}_{2}>1$-en tantos por ciento-, conforme a la norma española para cementos RC-75 (2). Su análisis químico completo se dio en (1).

\subsubsection{Puzolanas naturales}

Las utilizadas fueron pumitas y otras rocas volcánicas similares procedentes de las zonas central y nororiental del país. Su análisis químico está dado en (1).

\subsubsection{Cenizas volantes}

Las utilizadas fueron las mismas empleadas en la fabr:cación de algunos de los "cementos actuales" PA-350. Proceden de la zona noroeste del país y su composición química y otras características están asimismo dadas en (1).

\subsubsection{Filler}

En general el filler fue calizo de diferentes orígenes, con un contenido medio de $\mathrm{CaCO}_{3}$ en forma de calcita de un 90 por ciento, aproximadamente.

\subsection{Molienda de los cementos}

Los resultados obtenidos en la molienda de los cementos se muestran en la tabla 1-a, en la cual se dan detalles relativos a las proporciones e incrementos de las adiciones al pasar de los "cementos actuales" a los "cementos-proyecto". En la tabla 1-b se dan también los consumos de energía de molienda de las dos series, expresados en $\mathrm{kwh} / \mathrm{t}$ de cemento producido en cada caso.

Como se puede observar, el consumo de energía en la molienda de los "cementos-proyecto" P-450 y P-350 es en general más bajo que el de los correspondientes "cementos actuales", mientras que el consumo que corresponde a los "cementos-proyecto" PA-350 es mayor que el de los "cementos actuales" PA-350. Sin embargo, lo que importa, como se verá después (4.4.), es la estrecha relación existente entre la proporción de adición al cemento y el ahorro total de energía conseguido.

\subsection{Consumo de energía: clínker, adiciones, cemento}

De acuerdo con los valores expuestos en la tabla 2, el clínker requiere el mayor consumo de energia $\left(108,9 \mathrm{tep} / \mathrm{t} \times 10^{-3} ;\right.$ tep $=$ toneladas equivalentes de petróleo; $1 \mathrm{kwh}=0,247$ tep $\left.\times 10^{-3}\right)$, seguido de las puzolanas $(7,5)$, las escorias granuladas de horno alto $(6,9)$, las cenizas volantes $(5,6)$ y el filler calizo $(1,0)$. Por lo tanto, la sustitución parcial de clínker por adiciones es una solución económica y técnicamente conveniente.

En cuanto se refiere al consumo total de energía por los cementos, se puede observar que son posibles ahorros en todos los "cementos-proyecto" producidos, en comparación con los correspondientes "cementos actuales", a pesar de que los "cementos-proyecto" PA-350 exigen una cantidad mayor de $\mathrm{kwh} / \mathrm{t}$ para su molienda (4.3), pues la diferencia es más que compensada por el ahorro de calorias (tep) resultante de la sustitución parc:al de clínker por adiciones. 
Estos ahorros, expresados en tep/t $\times 10^{-3}$ son, en promedio, de 5,3, 6,0 y 12,1 para los cementos P-450, P-350 y PA-350, respectivamente. Las relaciones de estas cifras y las de la tabla 1-b para variaciones -incrementos- de las adiciones (de 5,1, 6,4 y 12,2 para los mismos cementos en cada caso) son de 1,04, 0,94 y 0,98 -en promedio total, 0,99-, lo cual significa que el ahorro obtenido al utilizar las adiciones es aproximadamente de 1 tep por cada $10 \mathrm{t}$ de materiales molidos conjuntamente con el clínker.

\subsection{Resultados del análisis químico y de los ensayos de resistencia según el Pliego RC-75}

Los valores medios de los resultados del análisis químico y de los ensayos de resistencia a 3, 7 y 28 días, tanto de los "cementos actuales" como de los "cementos-proyecto" P-450, P-350 y PA-350 — detalles en (1)—, muestran que:

i) las diferencias entre los "cementos actuales" y los "cementos proyecto" son evidentes en lo que se refiere a la pérdida al fuego y al res:duo insoluble;

ii) la calidad -resistencia mecánica- de los "cementos-proyecto" es semejante a la de los "cementos actuales" P-450 y P-350, y en algunos casos superior;

iii) en el caso de los cementos PA-350 no se observan sino diferencias muy pequeñas entre algunos "cementos-proyecto" y "cementos actuales", en favor de estos últimos.

En consecuencia, parece ser recomendable aprovechar el ahorro de energía que resulta de añadir 5 por ciento de filler calizo a los "cementos actuales" P-450 y P-350, así como elevar de 20 a 35 por ciento el contenido de adiciones a los "cementos actuales" PA-350.

\section{ENSAYOS ADICIONALES}

Habida cuenta de que los ensayos de resistencia descritos en lo que precede se habían llevado a cabo con probetas de mortero de relación agua/cemento constante -0,50 (2)-, podría parecer dudoso que el comportamiento de los "cementos-proyecto" fuese similar al de los correspondientes "cementos actuales", si unos y otros se ensayasen en otras condiciones distintas. Por lo tanto, se llevaron a cabo los ensayos adicionales siguientes - detallados en (1)-:

i) ensayo normal RC-75 (2): con mortero $1: 3$ y relación agua/cemento constante de 0,5 (probetas prismáticas de $4 \times 4 \times 16 \mathrm{~cm}$ y probetas cilíndricas de $10 \times \varnothing 5 \mathrm{~cm}$ );

ii) ensayo "110": con el mismo mortero de i), pero a escurrimiento constante -110 en la mesa de sacudidas-, con relación agua/cemento variable (probetas prismáticas de $4 \times 4 \times 16 \mathrm{~cm}$ );

iii) ensayo "110-U": con el mismo mortero de i) y ii), pero añadiendo un 5 por ciento de un material sílico-calcáreo (margo-arcilloso) extrafino a la arena normal utilizada (probetas prismáticas de $4 \times 4 \times 16 \mathrm{~cm}$ y cilíndricas de $10 \times \varnothing 5 \mathrm{~cm}$ ). El objeto de este ensayo fue el de acercar lo más posible las condiciones a las del "macro-ensayo" con probetas cilíndricas de $30 \times \varnothing 15 \mathrm{~cm}$, si bien sustituyendo el hormigón por mortero, y la medida de la consistencia mediante el cono Abrams por la de la mesa de sacudidas, así como el de estudiar la influencia del material extrafino añadido.

\subsection{Resultados con probetas prismáticas}

\subsubsection{Resultados del ensayo normal RC-75}

Este ensayo "patrón" ha permitido comparar los resultados de los otros ensayos, con ca- 
da uno de los "cementos-proyecto" y "cementos actuales" estudiados.

\subsubsection{Resultados del ensayo " $110 "$}

La relación agua/cemento de este ensayo es mayor que la del ensayo RC-75 (2), lo cual supuso que las resistencias medias del ensayo "110" resultantes a 90,180 y 365 días fueron menores, tanto para los "cementos-proyecto" como para los "cementos actuales" P-450, P-350 y PA-350, comparadas con las del ensayo normal RC-75.

\subsubsection{Resultados del ensayo "110-U"}

Las resistencias fueron mucho más bajas aún, en todos los casos, a causa de la gran exigencia de agua de los morteros, debida a su vez a la presencia del material extrafino. Algunos de los cementos ensayados mostraron una mayor sensibilidad a este efecto.

La tabla 3 resume los resultados de los tres ensayos a edades entre 3 y 365 días, de los cementos P-450, P-350 y PA-350. Los valores dados en ella son las relaciones entre las resistencias de los "cementos-proyecto" y las de los correspondientes "cementos actuales". Se puede observar que no existen prácticamente diferencias entre las resistencias resultantes para los "cementos-proyecto" y los "cementos actuales" P-450 y P-350, según los ensayos RC-75, "110" y "110-U”. Sin embargo, los "cementos-proyecto" PA-350 muestran una resistencia apreciablemente menor, según el ensayo "110-U".

\subsection{Resultados con probetas cilíndricas}

\subsubsection{Resultados del ensayo normal $R C-75$}

Estos resultados muestran que el simple camb:o de forma y dimensiones de las probetas causa una apreciable variación de resistencia, en comparación con los resultados del mismo ensayo efectuado con probetas prismáticas. Así sucede tanto para los "cementos-proyecto" como para los "cementos actuales" P-450, P-350 y PA-350. La tabla 4 presenta las relaciones entre las resistencias de probetas prismáticas y cilíndricas. El valor medio de todas las relaciones es prácticamente 81 , muy próximo al 80 por ciento, usual en este tipo de relación.

\subsubsection{Resultados del ensayo "110-U"}

Como en 5.1.3., las resistencias son mucho más afectadas por las altas relaciones agua/cemento, como consecuencia de la gran exigencia de agua por el mortero, debida a la presencia del 5 por ciento de material extrafino. La tabla 5 muestra las relaciones entre las resistencias de los "cementos-proyecto" y de los "cementos actuales" P-450, P-350 y PA-350 ensayados con probetas cilíndricas.

Por otra parte, la comparación de las tablas 3 y 5 permite establecer que:

i) los "cementos-proyecto" y los "cementos actuales" P-450 y P-350 sometidos tanto al ensayo RC-75 como al ensayo "110-U", muestran un comportamiento semejante;

ii) los cementos PA-350 en general, y particularmente los "cementos-proyecto" PA-350, son afectados más desfavorablemente por el ensayo "110-U".

Al tratar de interpretar este comportamiento diferente de los "cementos-proyecto" PA-350, la tabla 6-a muestra los porcentajes y la naturaleza de las adiciones utilizadas - parte izquierda-, así como las relaciones entre las resistencias de los "cementos-proyecto" y de los "cementos actuales" PA-350, según el ensayos RC-75 - parte derecha-. La tabla 6-b, 
con la misma parte izquierda, muestra en su parte derecha las relaciones entre las resistencias de los "cementos-proyecto" y de los "cementos actuales" PA-350, según el ensayo "110-U". Ambas tablas presentan también los ahorros de energía en cada caso, expresados en tep/t.

Los valores relativos al ensayo RC-75 (tabla 6-a) permiten inferir lo siguiente:

i) el efecto del filler calizo en las resistencias mecánicas es evidente;

ii) este efecto depende de la proporción de filler añadido, en esta forma:

a) porcentajes inferiores al 10 por ciento actúan favorablemente - al menos no perjudicialmente-, en general a cualquier edad (de las consideradas en este estudio);

b) porcentajes superiores al 10 por ciento actúan desfavorablemente, y tanto más cuanto mayor es la proporción de filler añadido y la edad de las probetas, de forma que el efecto beneficioso inicialmente observado se sustituye por una caída de resistencia -es decir, por un menor crecimiento de la resistencia a edades más largas-.

En cuanto a los valores de los ensayos "110-U" (tabla 6-b), las inferencias pueden ser las mismas, si bien el límite entre los efectos favorables y desfavorables se debe establecer en 5 por ciento, en lugar de 10 por ciento de filler calizo añadido.

\section{CONCLUSIONES}

Antes de establecer conclusiones es conveniente señalar que éstas podrán ser válidas con tal de que -y sólo si- los "cementos-proyecto" considerados aquí se producen en fábricas de cemento. Estas son las únicas que razonablemente poseen personal, equipo y medios de control para garantizar calidad, regularidad y seguridad, todo ello avalado a su vez por la responsabilidad. Dicho de otro modo, no es en absoluto recomendable (sino todo lo contrario) añadir cualquier material al cemento y/o al hormigón -salvo los aditivos para éste- in situ (en obra). Con tal de que esta condición se cumpla, las principales conclusiones de este trabajo podrían ser las siguientes:

1) El medio más inmediato fácil y económico de ahorrar energía en la fabricación de cemento sin afectar adversamente a la calidad del producto es la utilización de adiciones activas $\mathrm{y} / \mathrm{o}$ de fillers calizos.

2) Hasta un 5 por ciento de filler calizo añadido a los actuales cementos españoles P-450 y P-350 actúa favorablemente sobre las resistencias a cualquier edad.

3) El límite superior de 20 por ciento de adiciones activas en los cementos actuales PA-350 podría ser elevado hasta el 35 por ciento, incluyendo en él un 5 por ciento de filler calizo.

4) La fabricación de los correspondientes "cementos-proyecto" podría ahorrar energía en proporciones de un 4 a un 5 por ciento en los cementos P-450 y P-350, y de un 12 a un 13 por ciento en los cementos PA-350, sin cambio sustancial de la calidad, comparada con la correspondiente a los cementos actuales.

5) En general, la estimación del ahorro nacional de energía podría ser, en promedio, de 1 tonelada de fuel-oil por cada 10 toneladas de adiciones utilizadas. 
6) La relación agua/cemento y el tamaño y forma de las probetas, así como la presencia de materiales extrafinos añadidos al mortero influyen evidentemente en las resistencias de los cementos ensayados, y particularmente en las de los "cementos-proyecto" PA-350.

7) El ensayo RC-75 muestra que a cualquier edad entre 3 días y 1 año la resistencia de los "cementos-proyecto" P-450, P-350 y PA-350 es prácticamente el 100 por ciento de la resistencia de los correspondientes cementos actuales - variando los valores medios entre 95 y 102 por ciento, los valores individuales entre 87 y 108 por ciento, y siendo la media total de 98,5 por ciento-

8) Al añadir materiales activos o fillers calizos al clínker, las especificaciones sobre pérdida al fuego y residuo insoluble según RC-75 (2) dejan de tener sentido; además, no informan ni sobre la calidad del cemento ni sobre el comportamiento de éste, en la forma en que lo hacen, por ejemplo, las especificaciones sobre fraguado, resistencia, trabajabilidad, exigencia y retención de agua, estabilidad, durabilidad, etc.

9) La industria española del cemento -OFICEMEN (1)- considera aconsejable ahorrar energía en la fabricación del cemento, de acuerdo con las conclusiones 4) y 5), y es consciente de que para hacerlo es preciso el acuerdo de la Industria de la Construcción y de la Administración españolas. Hay que añadir que existe la intención de proseguir este estudio experimental por parte de la Industria del Cemento y de la Administración, haciéndolo extensivo a los hormigones reales de la práctica.

10) Finalmente, los ahorros en el consumo total de energía en las diferentes zonas de España, para cada tipo, clase y categoría de los cementos estudiados, se dan en la tabla-figura 7 , en la cual se exponen los valores individuales -en el mapa-, los intervalos - a la derecha- y el consumo total y el ahorro -abajo-.

Los autores firmantes de este trabajo, que representan a la Industria Española del Cemento a través de OFICEMEN, desean expresar su reconocimiento al CDTI -Centro de Desarollo Tecnológico Industrial (3), dependiente del MIE (Ministerio de Industria y Energía)-, por el apoyo dado a este trabajo (1), y particularmente al Prof. Dr. José CALLEJA, del IETCC, por sus observaciones, sugerencias y críticas al mismo, así como por la versión inglesa de él para su presentación en el Coloquio Internacional sobre Escorias y Cementos con adiciones, celebrado en Mons (Bélgica) del 7 al 11 de Septiembre de 1981.

\section{R E F E R E N C I A S}

(1) "Investigación del Ahorro de Energía en la Industria del Cemento". OFICEMEN : Agrupación de Fabricantes de Cemento de España, Madrid, 1981.

(2) "Pliego de Prescripciones Técnicas Generales para la Recepción de los Cementos: RC-75". Ministerio de Obras Públicas y Urbanismo: Madrid, 1975.

(3) "Situación Energética en la Industria. Sector CEMENTO". Ministerio de Industria y Energía. Centro de Estudios de la Energia, Madrid, 1979. 
T A B L A 1 -a

Adiciones y consumo de energía

\begin{tabular}{|c|c|c|c|c|c|c|c|c|c|c|c|c|c|c|}
\hline \multirow{3}{*}{\multicolumn{2}{|c|}{ CEMENTOS }} & \multirow{4}{*}{ 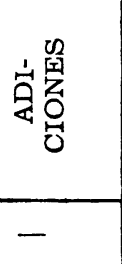 } & \multicolumn{10}{|c|}{$\mathrm{Z} \quad \mathrm{O} \quad \mathrm{N} A \mathrm{~A}$} & \multirow{2}{*}{\multicolumn{2}{|c|}{$\begin{array}{l}\text { Promedio } \\
\text { Nacional }\end{array}$}} \\
\hline & & & \multicolumn{2}{|c|}{1} & \multicolumn{2}{|c|}{2} & \multicolumn{2}{|c|}{3} & \multicolumn{2}{|c|}{4} & \multicolumn{2}{|c|}{5} & & \\
\hline & & & $\%$ & Varia- & $\%$ & & ic & Varia- & $\%$ & Varia- & $\%$ & Varia- & $\%$ & Varia- \\
\hline \multirow{2}{*}{$\begin{array}{l}\text { 10 } \\
\text { †' } \\
\text { م. }\end{array}$} & Actual & & 0 & & 0 & & 0 & & 0 & & 0 & & 0 & \\
\hline & Proy. & Filler & 5 & 5 & 4,9 & 4,9 & 5 & 5 & 5,4 & 5,4 & 5,2 & 5,2 & 5,1 & $+5,1$ \\
\hline \multirow{3}{*}{ 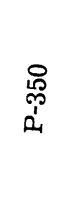 } & Actual & - & 0 & & 0 & & 0 & & 0 & & 0 & & 0 & \\
\hline & \multirow{2}{*}{ Proy. } & Escoria & - & & - & & - & & - & & 5 & & 1 & \\
\hline & & Filler & 5 & 5 & 5 & 5 & 5 & 5 & 4,8 & 4,8 & 7,5 & 7,5 & 5,4 & $+6,4$ \\
\hline \multirow{9}{*}{ 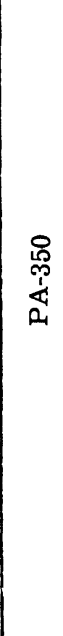 } & \multirow{4}{*}{ 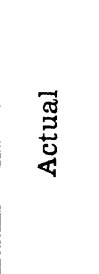 } & Escoria & - & & 16,8 & & - & & - & & 9,0 & & 5,2 & \\
\hline & & PUZ & 8,7 & & 1,9 & & 17,3 & & 13,6 & & - & & 8,3 & \\
\hline & & $\begin{array}{l}\text { Ceniza } \\
\text { volante }\end{array}$ & 1,6 & & - & & - & & 5,0 & & 6,4 & & 2,6 & \\
\hline & & Total & 10,3 & & 18,7 & & 17,3 & & 18,6 & & 15,4 & & 16,1 & \\
\hline & \multirow{5}{*}{ 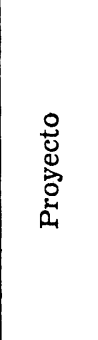 } & Escoria & - & & 19,2 & & - & & - & & 18,6 & & 7,6 & \\
\hline & & PUZ & 12,1 & & 2,0 & & - & & 15,7 & & - & & 5,8 & \\
\hline & & $\begin{array}{l}\text { Ceniza } \\
\text { volante }\end{array}$ & 4,6 & & - & & - & & 4,5 & & 9,6 & & 3,7 & \\
\hline & & Filler & 11,5 & & 11,7 & & 21,7 & & 7,9 & & 3,3 & & 11,2 & \\
\hline & & Total & 28,2 & 17,9 & 32,9 & 14,2 & 21,7 & 4,4 & 28,1 & 9,5 & 31,5 & 16,1 & 28,4 & $+12,3$ \\
\hline
\end{tabular}

TAB I, A 1 -b

Adiciones y consumo de energía

\begin{tabular}{|c|c|c|c|c|c|c|c|c|c|c|c|c|c|c|}
\hline \multirow{3}{*}{\multicolumn{2}{|c|}{ CEMENTOS }} & \multirow{3}{*}{ 宴罢 } & \multicolumn{10}{|c|}{$\begin{array}{lllll}Z & \mathrm{O} & \mathrm{A} & \mathrm{S}\end{array}$} & \multirow{2}{*}{\multicolumn{2}{|c|}{$\begin{array}{l}\text { Promedio } \\
\text { Nacional }\end{array}$}} \\
\hline & & & \multicolumn{2}{|c|}{1} & \multicolumn{2}{|c|}{2} & \multicolumn{2}{|c|}{3} & \multicolumn{2}{|r|}{4} & \multicolumn{2}{|r|}{5} & & \\
\hline & & & $\%$ & $\begin{array}{l}\text { Varia- } \\
\text { ción }\end{array}$ & $\%$ & $\begin{array}{l}\text { Varia- } \\
\text { ción }\end{array}$ & $\%$ & $\begin{array}{l}\text { Varia- } \\
\text { ción }\end{array}$ & $\%$ & $\begin{array}{l}\text { Varia- } \\
\text { ción }\end{array}$ & $\%$ & $\begin{array}{l}\text { Varia- } \\
\text { ción }\end{array}$ & $\%$ & $\begin{array}{c}\text { Varia- } \\
\text { ción }\end{array}$ \\
\hline \multicolumn{2}{|c|}{ Clinkeriz. } & $\mathrm{kg}$ fuel/t & 87,6 & & 90,9 & & 102,9 & & 99,1 & & 102,2 & & 96,5 & \\
\hline \multirow{6}{*}{ 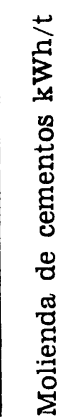 } & \multirow{2}{*}{ م. } & Actual & 81,8 & & 48,6 & & 60,0 & & 54,1 & & 52,0 & & 59,3 & \\
\hline & & Proyecto & 79,1 & $-2,7$ & 48,8 & $+0,2$ & 62,0 & $+2,0$ & 51,7 & $-2,4$ & 53,0 & $+1,0$ & 58,9 & $-0,4$ \\
\hline & \multirow{2}{*}{$\begin{array}{l}\text { 유 } \\
\text { p. } \\
\text { م⿱ }\end{array}$} & Actual & - & & 32,4 & & - & & 32,8 & & 37,3 & & 34,1 & \\
\hline & & Proyecto & - & & 32,3 & $-0,1$ & - & & 31,2 & $-1,6$ & 36,7 & $-0,6$ & 33,4 & $-0,7$ \\
\hline & \multirow{2}{*}{ 号 } & Actual & 39,1 & & 44,0 & 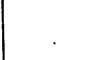 & 43,4 & & 42,0 & & 35,3 & & 40,7 & \\
\hline & & Proyecto & 43,1 & $+4,0$ & 50,3 & $+6,3$ & 50,2 & $+6,8$ & 51,1 & $+9,1$ & 44,5 & $+9,2$ & 47,8 & $+7,1$ \\
\hline
\end{tabular}


TABLA 2

Consumos de energía

\begin{tabular}{|c|c|c|c|c|c|c|c|c|c|c|c|c|c|c|}
\hline \multirow{3}{*}{\multicolumn{3}{|c|}{ MATERIALES }} & \multicolumn{10}{|c|}{$\begin{array}{lllll}\mathrm{Z} & \mathrm{O} & \mathrm{A} & \mathrm{S}\end{array}$} & \multirow{2}{*}{\multicolumn{2}{|c|}{$\begin{array}{l}\text { Promedios } \\
\text { Nacionales }\end{array}$}} \\
\hline & & & \multicolumn{2}{|c|}{1} & \multicolumn{2}{|c|}{2} & \multicolumn{2}{|c|}{3} & \multicolumn{2}{|c|}{4} & \multicolumn{2}{|r|}{5} & & \\
\hline & & & $\begin{array}{l}\mathrm{tep} / \mathrm{t} \\
\times 10^{-3}\end{array}$ & $\begin{array}{l}\text { Varia- } \\
\text { ción }\end{array}$ & $\begin{array}{l}\mathrm{tep} / \mathrm{t} \\
\times 10^{-3}\end{array}$ & $\begin{array}{l}\text { Varia- } \\
\text { ción }\end{array}$ & $\begin{array}{l}\mathrm{tep} / \mathrm{t} \\
\times 10^{3}\end{array}$ & $\begin{array}{l}\text { Varia- } \\
\text { ción }\end{array}$ & $\begin{array}{l}\mathrm{tep} / \mathrm{t} \\
\times 10^{-3}\end{array}$ & $\begin{array}{l}\text { Varia- } \\
\text { ción }\end{array}$ & $\begin{array}{l}\mathrm{tep} / \mathrm{t} \\
\times 10^{-3}\end{array}$ & $\begin{array}{c}\text { Varia- } \\
\text { ción }\end{array}$ & $\begin{array}{l}\mathrm{tep} / \mathrm{t} \\
\times 10^{-3}\end{array}$ & $\begin{array}{l}\text { Varia- } \\
\text { ción }\end{array}$ \\
\hline \multirow{6}{*}{\multicolumn{3}{|c|}{$\begin{array}{l}\text { Clinker } \\
\text { Yeso } \\
\text { Escorias } \\
\text { Puzolanas } \\
\text { Cenizas } \\
\text { Filler }\end{array}$}} & 100,2 & & 100,8 & & 111,5 & & 111,9 & & 112,9 & & 108,9 & \\
\hline & & & 1,5 & & 1,2 & & 3,0 & & 0,4 & & 4,0 & & 2,0 & \\
\hline & & & - & & 2,1 & & - & & - & & 11,8 & & 6,9 & \\
\hline & & & 5,7 & & - & & 12,2 & & 4,5 & & - & & 7,5 & \\
\hline & & & 4,5 & & - & & 一 & & 9,4 & & 2,9 & & 5,6 & \\
\hline & & & 0,7 & & 0,6 & & 2,2 & & 0,7 & & 0,8 & & 1,0 & \\
\hline \multirow{6}{*}{ 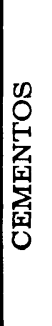 } & 学 & Actual & 112,0 & & 109,9 & & 108,4 & & 115,1 & & 113,7 & & 111,8 & \\
\hline & D & Proyecto & 106,7 & $-5,3$ & 104,6 & $-5,3$ & 103,6 & $-4,8$ & 109,1 & $-6,0$ & 108,4 & $-5,3$ & 106,5 & $-5,3$ \\
\hline & 용 & Actual & - & & 112,4 & & - & & 119,0 & & 114,4 & & 115,3 & \\
\hline & 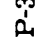 & Proyecto & - & & 107,3 & $-5,1$ & - & & 113,2 & $-5,8$ & 107,3 & $-7,1$ & 109,3 & $-6,0$ \\
\hline & 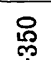 & Actual & 92,5 & & 92,6 & & 110,8 & & 96,0 & & 97,3 & & 97,8 & \\
\hline & 宾 & Proyecto & 76,7 & $-15,8$ & 79,8 & $-12,8$ & 99,9 & $-10,9$ & 87,7 & $-8,3$ & 84,2 & $-13,1$ & 85,7 & $-12,1$ \\
\hline
\end{tabular}

T A B L A 3

Relaciones entre resistencias de "cementos-proyecto" $y$ "cementos actuales"

\begin{tabular}{|c|c|c|c|c|c|c|c|c|c|}
\hline \multirow{2}{*}{ Edades } & \multicolumn{4}{|c|}{ P-450 } & \multicolumn{3}{c|}{ P-350 } & \multicolumn{3}{c|}{ PA-350 } \\
\cline { 2 - 11 } & $\begin{array}{c}\text { Ensayo } \\
\text { RC-75 }\end{array}$ & $\begin{array}{c}\text { Ensayo } \\
110\end{array}$ & $\begin{array}{c}\text { Ensayo } \\
110-U\end{array}$ & $\begin{array}{c}\text { Ensayo } \\
\text { RC-75 }\end{array}$ & $\begin{array}{c}\text { Ensayo } \\
110\end{array}$ & $\begin{array}{c}\text { Ensayo } \\
110-U\end{array}$ & $\begin{array}{c}\text { Ensayo } \\
\text { RC-75 }\end{array}$ & $\begin{array}{c}\text { Ensayo } \\
110\end{array}$ & $\begin{array}{c}\text { Ensayo } \\
110-U\end{array}$ \\
\hline $3 \mathrm{~d}$ & 104,7 & 102,4 & 95,1 & 93,4 & 99,4 & 94,0 & 107,8 & 107,9 & 92,5 \\
\hline $7 \mathrm{~d}$ & 103,0 & 99,0 & 93,3 & 97,0 & 98,4 & 92,4 & 99,6 & 96,7 & 87,1 \\
\hline $28 \mathrm{~d}$ & 100,0 & 96,1 & 92,5 & 94,9 & 95,9 & 93,6 & 93,6 & 89,4 & 86,7 \\
\hline $90 \mathrm{~d}$ & 100,2 & 95,9 & 93,4 & 94,0 & 92,4 & 96,4 & 95,3 & 88,2 & 86,9 \\
\hline $180 \mathrm{~d}$ & 99,3 & 94,9 & 92,7 & 93,8 & 91,8 & 94,5 & 95,1 & 90,2 & 87,2 \\
\hline $365 \mathrm{~d}$ & 99,8 & 97,9 & 92,4 & 94,7 & 90,9 & 94,2 & 96,7 & 88,6 & 86,6 \\
\hline$\overline{\mathrm{X}}$ & 101,1 & 97,7 & 93,2 & 94,6 & 94,8 & 94,1 & 98,0 & 93,5 & 87,8 \\
\hline
\end{tabular}

TABLA 4

Relaciones entre resistencias $R C-75$ (probetas cilíndricas) y RC-75 (probetas prismáticas)

\begin{tabular}{|c|l|c|c|c|c|c|c|c|}
\hline \multicolumn{2}{|c|}{ Cementos } & $3 \mathrm{~d}$ & $7 \mathrm{~d}$ & $28 \mathrm{~d}$ & $90 \mathrm{~d}$ & $180 \mathrm{~d}$ & $365 \mathrm{~d}$ & $\overline{\mathrm{X}}$ \\
\hline \multirow{3}{*}{ P-450 } & Actual & 82,2 & 81.8 & 80,8 & 78,8 & 79,4 & 78,6 & 80,2 \\
\cline { 2 - 10 } & Proyecto & 78,6 & 82,6 & 81,6 & 76,3 & 80,6 & 79,9 & 79,9 \\
\hline \multirow{3}{*}{ P-350 } & Actual & 84,8 & 82,5 & 81,2 & 79,2 & 84,3 & 75,5 & 81,2 \\
\cline { 2 - 10 } & Proyecto & 77,7 & 82,2 & 84,2 & 85,9 & 84,5 & 78,9 & 82,2 \\
\hline \multirow{3}{*}{ PA-350 } & Actual & 82,1 & 81,8 & 85,0 & 80,6 & 82,8 & 79,6 & 82,0 \\
\cline { 2 - 10 } & Proyecto & 84,0 & 84,4 & 80,6 & 79,7 & 80,2 & 75,7 & 80,8 \\
\hline
\end{tabular}


T A B L A 5

Relaciones entre resistencias de "cementos-proyecto" $y$ "cementos actuales" ensayados en probetas cilindricas

\begin{tabular}{|c|c|c|c|c|c|c|}
\hline \multirow{2}{*}{ CEMENTOS } & \multicolumn{2}{|c|}{ P-450 } & \multicolumn{2}{c|}{ P-350 } & \multicolumn{2}{c|}{ PA-350 } \\
\hline Eciades Ensayos & RC-75 & $110-\mathrm{U}$ & $\mathrm{RC}-75$ & $110-\mathrm{U}$ & $\mathrm{RC}-75$ & $110-\mathrm{U}$ \\
\hline $3 \mathrm{~d}$ & 100,3 & 92,1 & 87,5 & 92,9 & 105,0 & 89,7 \\
\hline $7 \mathrm{~d}$ & 103,2 & 100,3 & 101,7 & 99,5 & 107,4 & 82,6 \\
\hline $28 \mathrm{~d}$ & 101,3 & 97,3 & 99,4 & 93,8 & 93,5 & 93,7 \\
\hline $90 \mathrm{~d}$ & 99,5 & 98,7 & 102,0 & 100,2 & 97,0 & 89,9 \\
\hline $180 \mathrm{~d}$ & 102,4 & 96,0 & 97,7 & 92,4 & 95,0 & 92,5 \\
\hline $365 \mathrm{~d}$ & 104,5 & 95,2 & 102,3 & 96,1 & 94,0 & 96,5 \\
\hline
\end{tabular}

TABLA $6-\mathrm{a}$

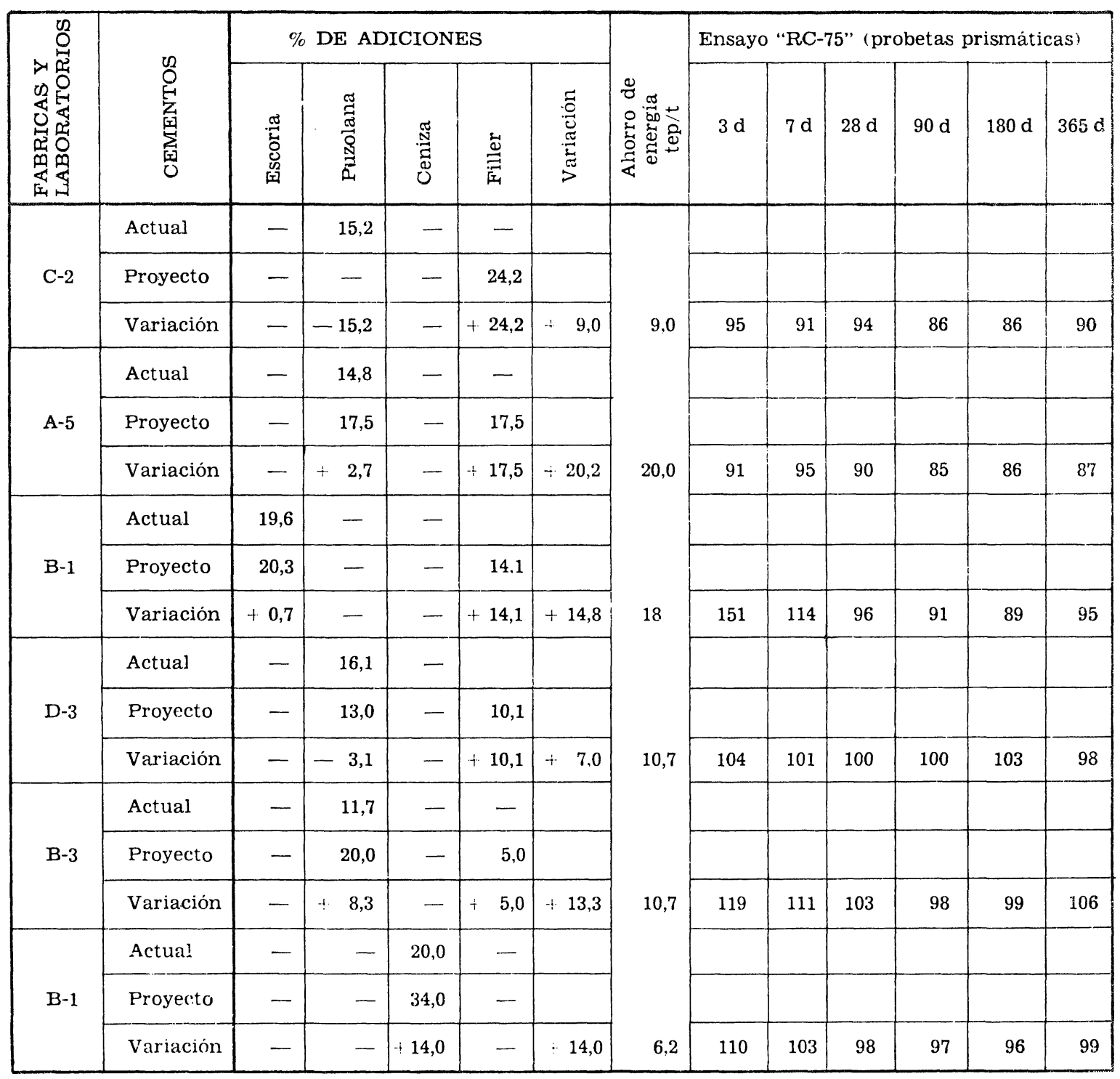


TABLA $6-\mathrm{b}$

\begin{tabular}{|c|c|c|c|c|c|c|c|c|c|c|c|c|c|}
\hline \multirow{2}{*}{ 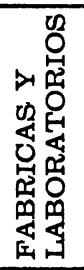 } & \multirow[b]{2}{*}{ 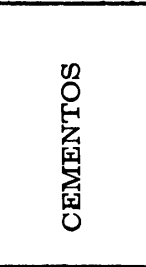 } & \multicolumn{5}{|c|}{$\%$ DE ADICIONES } & \multirow[b]{2}{*}{ 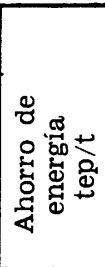 } & \multicolumn{6}{|c|}{ Ensayo "110-U" (probetas cilindricas) } \\
\hline & & 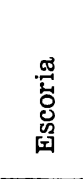 & 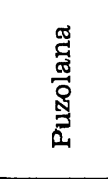 & 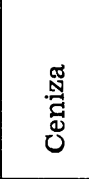 & 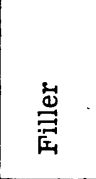 & 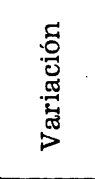 & & $3 d$ & $7 \mathrm{~d}$ & $28 \mathrm{~d}$ & $90 d$ & $180 \mathrm{~d}$ & $365 d$ \\
\hline \multirow{3}{*}{$\mathrm{C}-2$} & Actual & - & 15,2 & - & - & & \multirow{5}{*}{9,0} & & & & & & \\
\hline & Proyecto & - & - & - & 24,2 & & & & & & & & \\
\hline & Variación & - & $-15,2$ & - & $+24,5$ & $+9,0$ & & 89 & 82 & 96 & 84 & 80 & 82 \\
\hline \multirow{3}{*}{ A-5 } & Actual & - & 14,8 & - & - & & & & & & & & \\
\hline & Proyecto & - & 17,5 & - & 17,5 & & & & & & & & \\
\hline & Variación & - & $+2,7$ & - & $+17,5$ & $+20,2$ & \multirow[t]{2}{*}{20,0} & 75 & 74 & 77 & 73 & 74 & 81 \\
\hline \multirow{3}{*}{ B-1 } & Actual & 19,6 & - & - & & & & & & & & & \\
\hline & Proyecto & 20,3 & - & - & 14,1 & & \multirow{4}{*}{18} & & & & & & \\
\hline & Variación & $+0,7$ & - & - & $+14,1$ & $+14,8$ & & 105 & 90 & 87 & 82 & 82 & 83 \\
\hline \multirow{3}{*}{$D-3$} & Actual & - & 16,1 & - & & & & & & & & & \\
\hline & Proyecto & - & 13,0 & - & 10,1 & & & & & & & & \\
\hline & Variación & - & $-3,1$ & - & $+10,1$ & $+7,0$ & \multirow[t]{2}{*}{10,7} & 72 & 69 & 79 & 80 & 82 & 90 \\
\hline \multirow{3}{*}{ B-3 } & Actual & - & 11,7 & - & - & & & & & & & & \\
\hline & Proyecto & - & 20,0 & - & 5,0 & & \multirow{5}{*}{10,7} & & & & & & \\
\hline & Variación & - & $+8,3$ & - & $+\mathbf{5 , 0}$ & $+13,3$ & & 107 & 92 & 104 & 100 & 107 & 108 \\
\hline \multirow{3}{*}{ B-1 } & Actual & - & - & 20,0 & - & & & & & & & & \\
\hline & Proyecto & - & - & 34,0 & - & & & & & & & & \\
\hline & Variación & - & - & $+14,0$ & - & $+14,0$ & & 108 & 101 & 92 & 105 & 94 & 101 \\
\hline
\end{tabular}



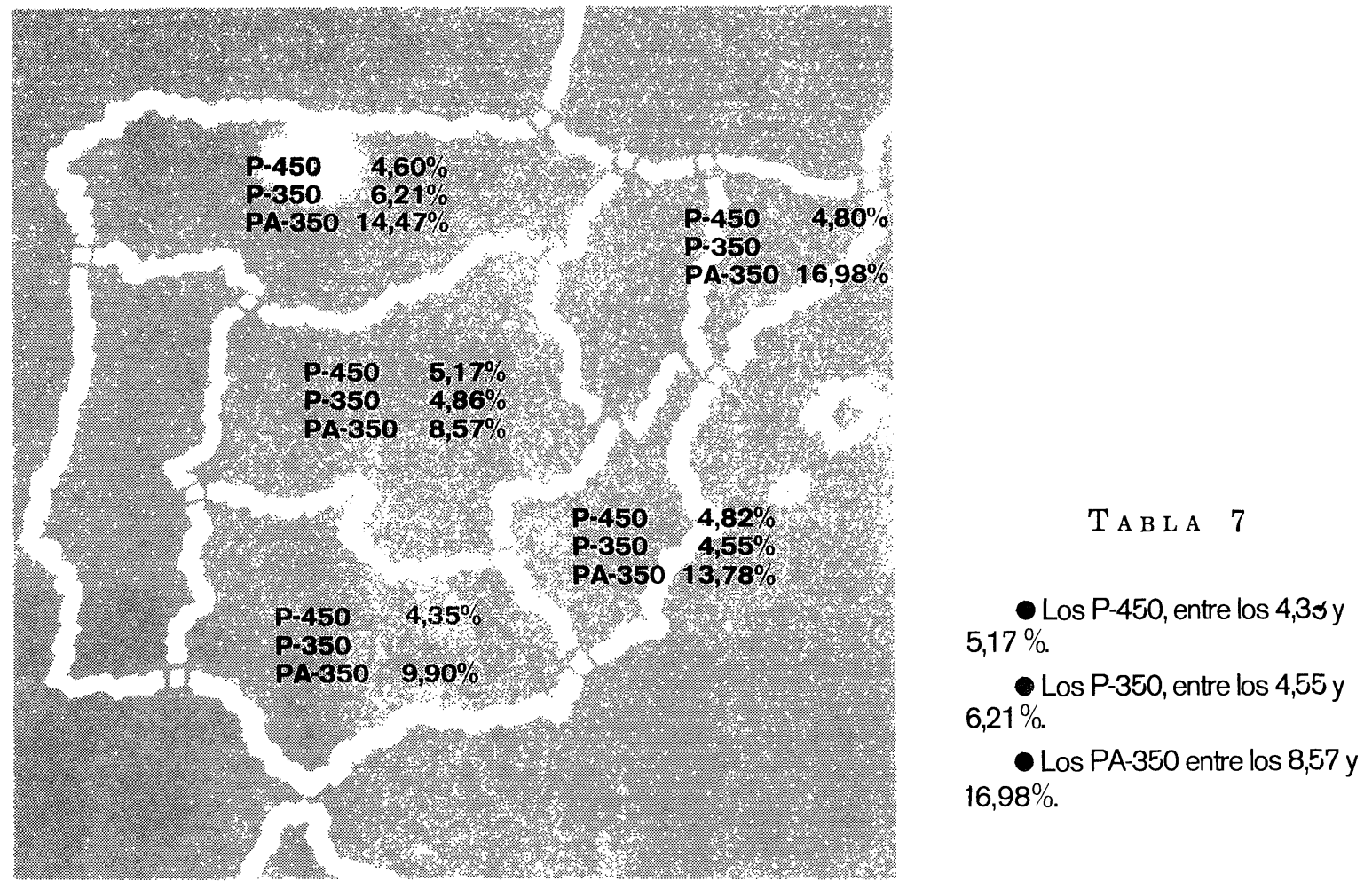

\begin{tabular}{|c|c|}
\hline $\begin{array}{c}\text { CONSUMO TOTAL } \\
\text { Tep. }\end{array}$ & AHORRO \\
1.978 & SEGUN RESULTADOS \\
\hline 3.350 .307 & 238.649 \\
$100 \%$ & $\mathbf{7 , 1} \%$ \\
\hline
\end{tabular}

Materiales De Construccion N.` $183-1981$ 